\title{
Rydberg states and Molecular Photo- Absorption Spectrum of Vinyl Chloride
}

\author{
Anwar Mustafa Ezzat Al-Faydhi \\ Physics Dept./College of Science/Mosul University
}

Received

17/10/2006
Accepted

14/11/2006

\begin{abstract}
المخلص

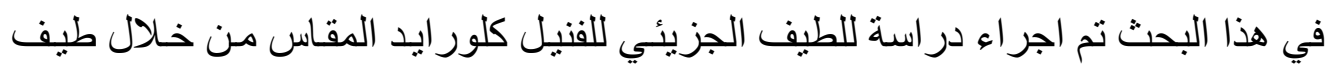

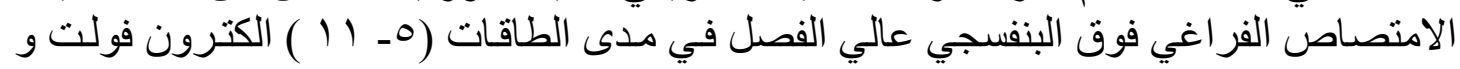

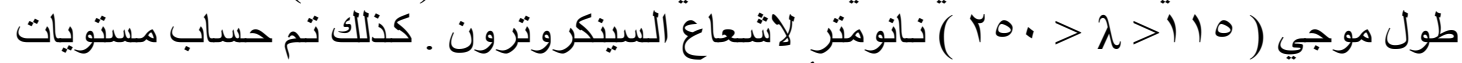

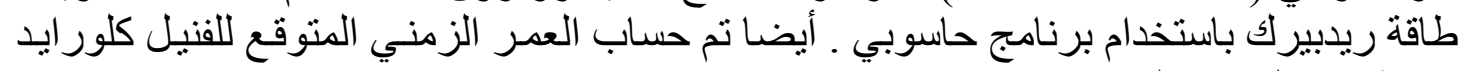

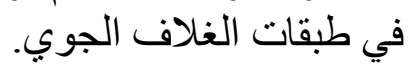

\begin{abstract}
In this work, a comprehensive study of the molecular spectroscopy of vinyl chloride is reported. A high resolution VUV photo absorption spectrum of vinyl chloride has been measured using a synchrotron radiation in the range $(5-11) \mathrm{eV}(115>\lambda>250) \mathrm{nm}$. The excited Rydberg states of vinyl chloride were calculated using a software program. The photolysis has also been investigated to give an estimate of the life time in the atmosphere.
\end{abstract}

\section{Introduction}

Research in the area of molecular excited electronic states covers a broad field of endeavour, touching as it does many aspects in spectroscopy, dynamics/kinetics, photo-dissociation, and chemical reactions (1). Vinyl Chloride is a highly volatile molecule that exists as a gas at Standard Temperature and Pressure; for this reason, occupational exposure to Vinyl Chloride Molecule (VCM) occurs primarily through inhalation.

Vinyl chloride is most commonly released into the environment as gas emissions or wastewater from polyvinyl chloride (PVC) plastic manufacturing plants. Liquid vinyl chloride evaporates easily into the air. Vinyl chloride, if it is near the surface of soil or water, can also evaporate.

Vinyl chloride in the air can break down within a few days to other substances, some of which can be harmful. Small amounts of vinyl chloride can dissolve in water. Vinyl chloride formed from the 
breakdown of other chemicals can enter groundwater. Vinyl chloride is unlikely to build up in plant, animal, or aquatic life. The independent scientific community has recognized VCM as a multi-potential carcinogen for almost thirty years and it is recognized as a potent cause of a wide variety of cancers arising in various organs of the body, both in humans and in animals. These cancers are not at all limited to vinyl chloride's "signal neoplasm," an giosarcoma of the liver, whose very existence in a given population strongly implies a larger occupational cancer risk caused by vinyl chloride. Most vinyl chloride is used to make polyvinyl chloride (PVC) plastic and vinyl products (2).

Acute (short-term) exposure to high levels of vinyl chloride in air has resulted in central nervous system effects (CNS), such as dizziness, drowsiness, and headaches in humans. Chronic (long-term) exposure to vinyl chloride through inhalation and oral exposure in humans has resulted in liver damage. Cancer is a major concern from exposure to vinyl chloride via inhalation, as vinyl chloride exposure has been shown to increase the risk of a rare form of liver cancer in humans. It has been classified vinyl chloride as a Group A, human carcinogen.

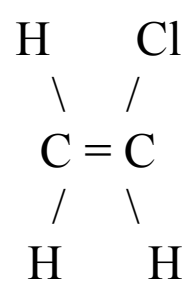

Fig. ( 1 )The molecular structure of Vinyl chloride $\left(\mathrm{C}_{2} \mathrm{H}_{3} \mathrm{Cl}\right)$

Despite being an atmospheric pollutant, vinyl chloride $\sim \mathrm{VC}$, $\mathrm{C}_{2} \mathrm{H}_{3} \mathrm{Cl}$ ) has been widely used in the industry for poly $\sim$ vinyl chloride production (3). The understanding of its photochemistry and spectroscopy is therefore very important. The purpose of this experiment is to measure cross-section for the photo ionisation of ions as a function of photon energy, and also to determine the Rydberg states for the molecule vinylchloride through the use of Synchrotron radiation. The Syncrotron radaition provides an excellent source of photons for photo absorption experiments. The emission is entense and extends over a contiuous energy range. Further more the light is highly colimated and completely polarised in the plane of the stoage ring (4).

The emission of synchrotron radiation is the product of the acceleration of charged particle. In a synchrotron radiation source (SRS), the charged particles are electrons and the key acceleration is initiated by strong magnetic fields. The applied magnetic fields are perpendicular to 
the pulse velocity so electrons fellow arced trajectories. The intensity of the radiation output of an SRS is proportional to the square of the electron velocity.

\section{Theory}

The features of the spectra can be labelled by calculated assignments representing Rydberg series. These calculations are taken place from the Rydberg formula:

$$
E_{n}=I . E .-\frac{R}{(n-\delta)^{2}}
$$

Where $E_{n}$ is the energy of the experimental band of the spectrum , I.E. is the ionization limit of the series , $\mathrm{R}$ is the Rydberg constant , $\mathrm{n}$ is the principle quantum number, and $\delta$ is the quantum defect which assigned in this work. However, it is also possible that Rydberg transitions may not be sharp and therefore not easily fit to the above equation. Broad transitions might also be valence transitions, and Robin notes that researchers at one time had a propensity to assume that any broad transition was a valence transition. In the vacuum-ultraviolet, valence and Rydberg states are much more likely to mix than they are in the quartz-ultraviolet. Rydberg states can be defined as those which fit Eq. (1) and whose wave functions are appropriate to generate such a series. Excitation energies are often reported as the difference between the Rydberg state and the corresponding ionization limit; these are referred to as \term values" in this context. For theoretical work, it may be more desirable to compute these term values than the actual excitation energies because the Rydberg state should look more like the ion than the ground state; orbital relaxation and correlation effects are more likely to cancel. While any particular valence transition may also fit Eq. (1), it will not belong to a series with increasing quantum number $\mathrm{n}$. An electron in a polyatomic molecule will fit such a formula if it is very diffuse, so that the core appears as a point charge. Of course this will never be entirely true, so the parameter \pm is added to correct for the extent to which the distant electron penetrates the core. The ground state of an atom may have an electron with a large radius and may thus be referred to as a Rydberg state; for neutral polyatomic, this never happens. Rydberg transitions may be split by core asymmetries, but the splittings decrease with increasing $\mathrm{n}$ because the core becomes better modelled as a point charge as the electron radius increases. For second-row atoms and molecules, the general trends in $\delta$ are:

ns: $\quad$ Penetrate the core to a large extent; $\delta \sim 1$. 
np: Penetrate to a moderate extent; $\delta \sim 0.6$.

nd: Hardly penetrate the core; $\delta \sim 0$.

For lower rows of the periodic table, more general rules are required. A Rydberg orbital will be non penetrating $(\delta \sim 0)$ if the core does not contain any occupied orbitals of its symmetry. Mulliken refers to such core orbitals as "real precursors". Hence $\delta \sim 0$ for, e.g., $2 \mathrm{p}, 3 \mathrm{~d}$, and 4f. (5)

\section{Experimental Apparatus}

The experiment was held in the Daresbury Synchrotron Radiation Source (SRS) fig. (2). A synchrotron is a source of radiation that provides a continuum of photons from infrared to X-ray energies. The main features of a synchrotron emission spectrum are that the intensities are many orders of magnitude higher than conventional light sources ( 8 ).

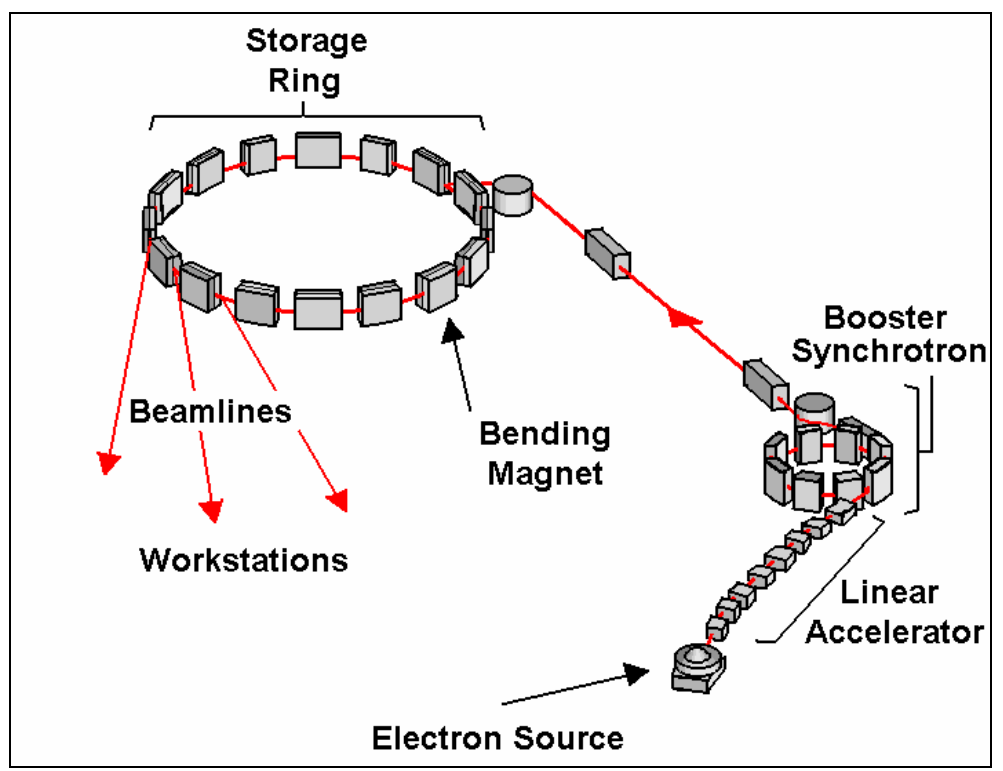

Fig.( 2 ) Schematic layout of the Daresbury SRS

The SRS offers a large mix of experimental facilities which deliver radiation with wavelengths extending from the infrared to hard X-rays. Synchrotron radiation is a very efficient source of light due to its high beam brilliance and excellent collimation. It has been previously applied as radiation source to the study of photochemical reactions on polymers (6). The SRS is a $2 \mathrm{GeV}$ electron storage ring, operated solely for the 
provision of synchrotron radiation (synchrotron light) for multiple simultaneous user experiments. It routinely operates at high circulating currents (150-250mA) and long lifetimes ( $>24$ hours), typically providing 4,500 hours per year of user beam with outstanding stability and reproducibility. The UV beam, fig. ( 3 ), passes through a simple static gas cell with an absorption path length of $25 \mathrm{~cm}$ and the transmitted intensity $\mathrm{I}_{\mathrm{t}}$ measured at $0.05 \mathrm{~nm}$ intervals using a photo multiplayer detector ( 500 Volt operating voltage ). The transmitted light intensity, as well as the sample pressure ( measured on an MKS 390HA Baratron capacitance manometer $\pm 0.1 \%)$.

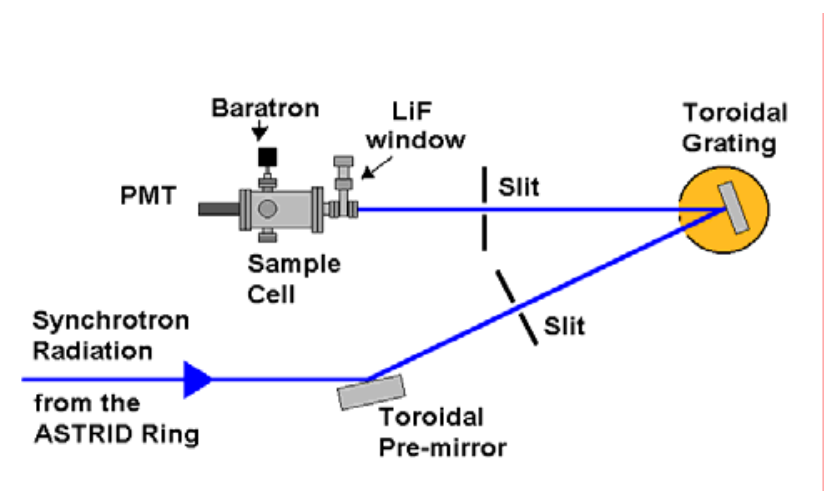

Fig. (3) Schematic diagram of the VUV beam line

The typical resolution was $\sim 0.075 \mathrm{~nm}$. The sample cell is then evacuated and the radiation transmitted through the empty cell $I_{0}$ is recorded. The absolute photo absorption cross section was calculated using the attenuation Beer - Lambert law :

$$
I=I_{o} \exp (-n \sigma x)
$$

With $\mathrm{n}$ is the target gas number density, $\sigma$ is the absolute photo absorption cross section of the molecular species and $\mathrm{x}$ is the path length (7).

\section{The Results}

The VUV photo absorption cross section for Vinyl Chloride $\left(\mathrm{C}_{2} \mathrm{H}_{3} \mathrm{Cl}\right)$ using special program $(9$ and 10$)$ is shown in fig. (4). 


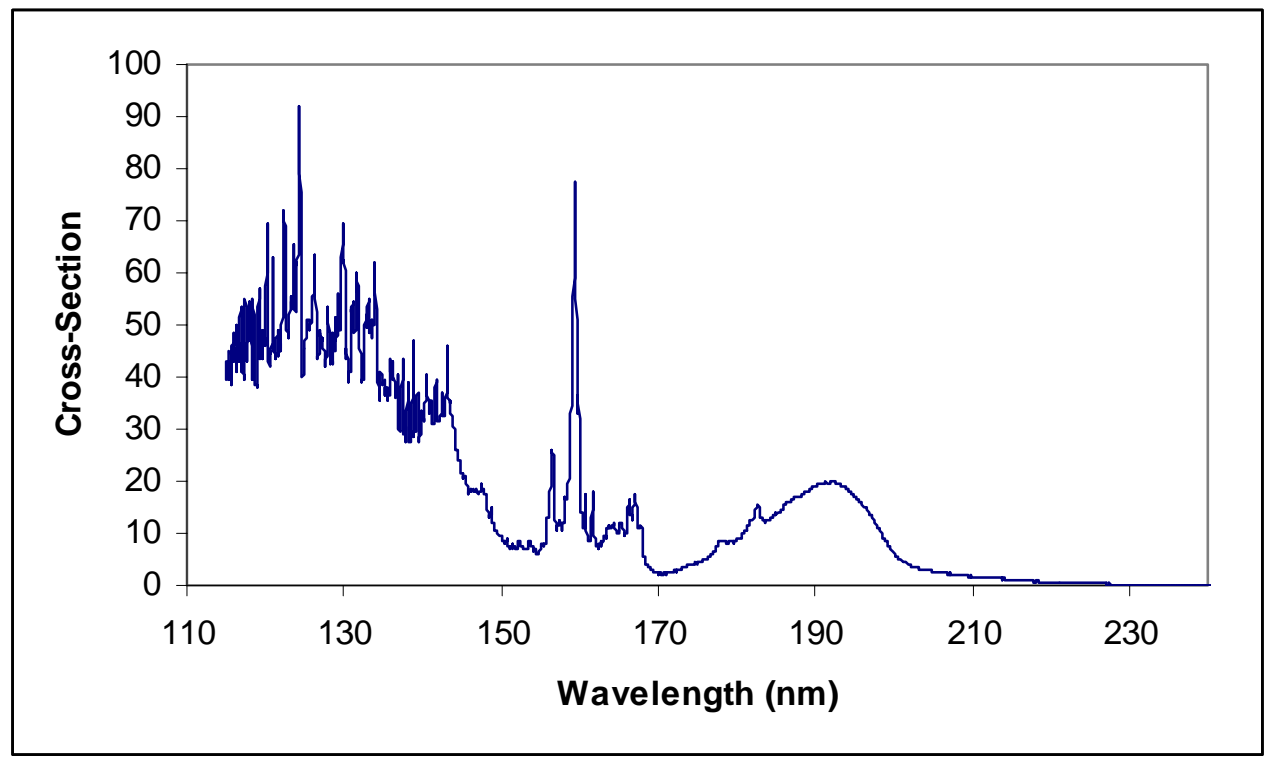

Fig. ( 4 ) The VUV photo absorption cross section for Vinyl Chloride

The spectrum feature a large number of fine structure, these structure have been tentatively assigned to members of Rydberg series converging to the ionization energy ( $9.99 \mathrm{eV})$ as in table ( 1$)$, using equation (1).

The assignments of the Rydberg states for Vinyl Chloride were calculated using a special software program written for this research.

\begin{tabular}{|c|c|}
\hline $\begin{array}{c}\text { Energy of the experimental } \\
\text { band of spectrum }\end{array}$ & Assignment \\
\hline 6.782 & $3 \mathrm{~s}$ \\
\hline 6.930 & $3 \mathrm{~s}$ \\
\hline 7.415 & $3 \mathrm{p}$ \\
\hline 7.453 & $3 \mathrm{p}$ \\
\hline 7.493 & $3 \mathrm{p}$ \\
\hline 7.667 & $3 \mathrm{p}$ \\
\hline 7.710 & $3 \mathrm{p}$ \\
\hline 7.778 & $3 \mathrm{~d}$ \\
\hline 7.867 & $3 \mathrm{~d}$ \\
\hline 7.919 & $3 \mathrm{~d}$ \\
\hline 8.061 & $3 \mathrm{~d}$ \\
\hline 8.132 & $3 \mathrm{~d}$ \\
\hline
\end{tabular}




\begin{tabular}{|c|c|}
\hline $\begin{array}{l}\text { Energy of the experimental } \\
\text { band of spectrum }\end{array}$ & Assignment \\
\hline 8.221 & $3 \mathrm{~d}$ \\
\hline 8.329 & $3 \mathrm{~d}$ \\
\hline 8.400 & $3 d$ \\
\hline 8.661 & $4 \mathrm{~s}$ \\
\hline 8.703 & $4 \mathrm{~s}$ \\
\hline 8.746 & $4 p$ \\
\hline 8.786 & $4 p$ \\
\hline 8.821 & $4 \mathrm{p}$ \\
\hline 8.843 & $4 p$ \\
\hline 8.897 & $4 p$ \\
\hline 8.935 & $4 \mathrm{~d}$ \\
\hline 8.977 & $4 d$ \\
\hline 9.020 & $4 d$ \\
\hline 9.063 & $4 \mathrm{~d}$ \\
\hline 9.096 & $4 d$ \\
\hline 9.119 & $4 d$ \\
\hline 9.129 & $4 \mathrm{~d}$ \\
\hline 9.177 & $5 \mathrm{~s}$ \\
\hline 9.201 & $5 \mathrm{~s}$ \\
\hline 9.256 & $5 p$ \\
\hline 9.315 & $5 p$ \\
\hline 9.414 & $5 d$ \\
\hline 9.457 & $6 s$ \\
\hline 9.551 & $6 \mathrm{~d}$ \\
\hline 9.562 & $6 \mathrm{~d}$ \\
\hline 9.614 & $7 \mathrm{~s}$ \\
\hline 9.641 & $7 p$ \\
\hline 9.686 & $7 d$ \\
\hline 9.758 & $8 \mathrm{~d}$ \\
\hline 9.816 & $9 d$ \\
\hline 9.871 & $11 \mathrm{~d}$ \\
\hline
\end{tabular}

Table (1) : Energy values and assignments of Rydberg series for Vinyl Chloride

The spectroscopic study of Rydberg states (illustrated in fig. (5)) helps us to illuminate the photo dissociation path ways and to manifest the complicated chemical-reaction mechanisms due to the multidimensionality in polyatomic molecular potential energy surfaces (1). 

J .Edu. Sci , Vol. (19) No.(2) 2007

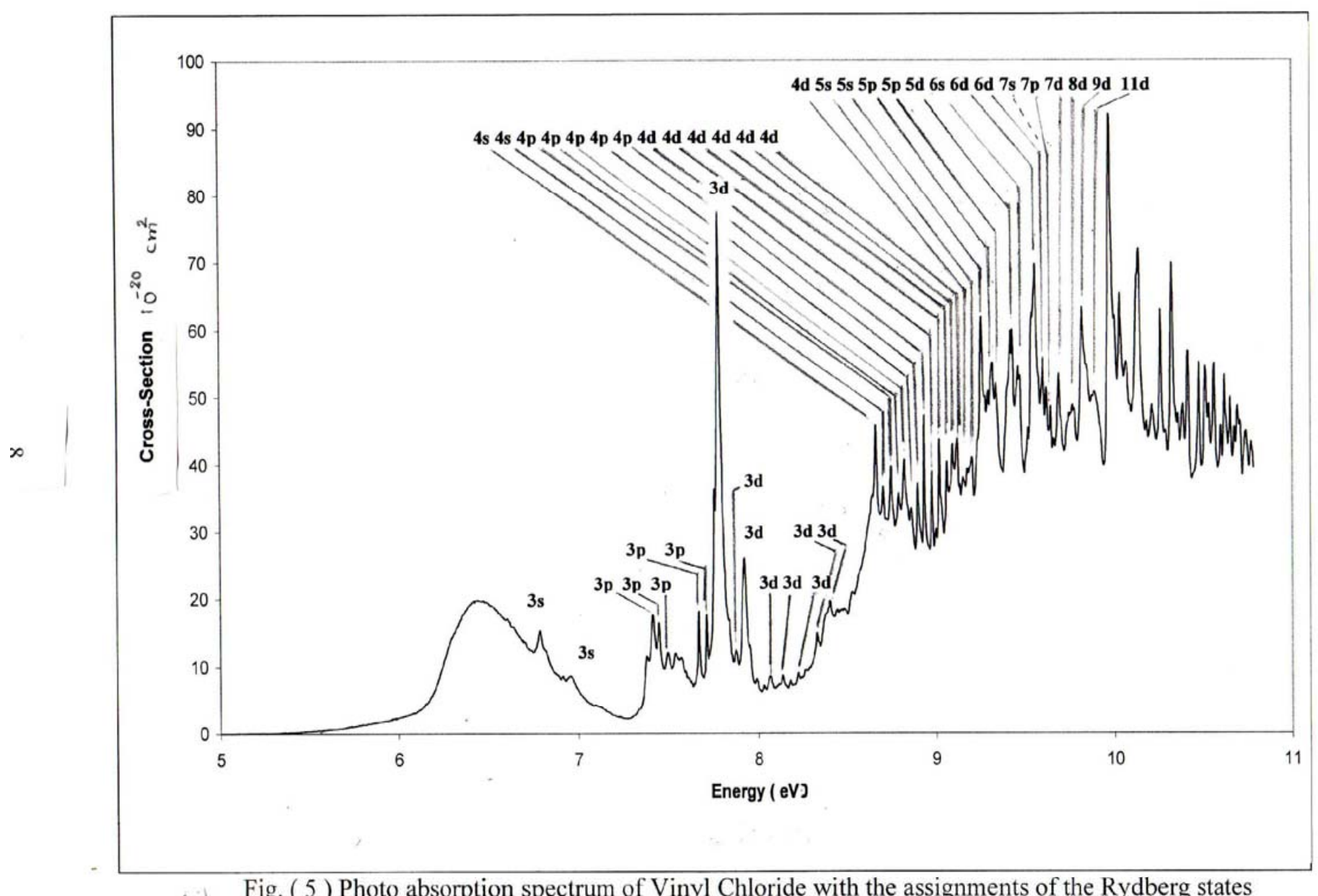

\begin{tabular}{ll}
\hline \hline & 46
\end{tabular} 



\section{Photolysis rate and local life time}

Using the absolute photo - absorption cross-sections the photolysis rates for Vinyl chloride can be calculated at $1 \mathrm{~km}$ altitude steps up to stratopause $(50 \mathrm{~km})$. Photolysis rate were calculated as the product of the solar actinic flux at a given wavelength and altitude, and the molecular photo absorption cross-section at the same wavelength for Vinyl chloride. At each altitude the total rates shown in fig. ( 6 ) are the summing over the individual photolysis rates for that altitude.

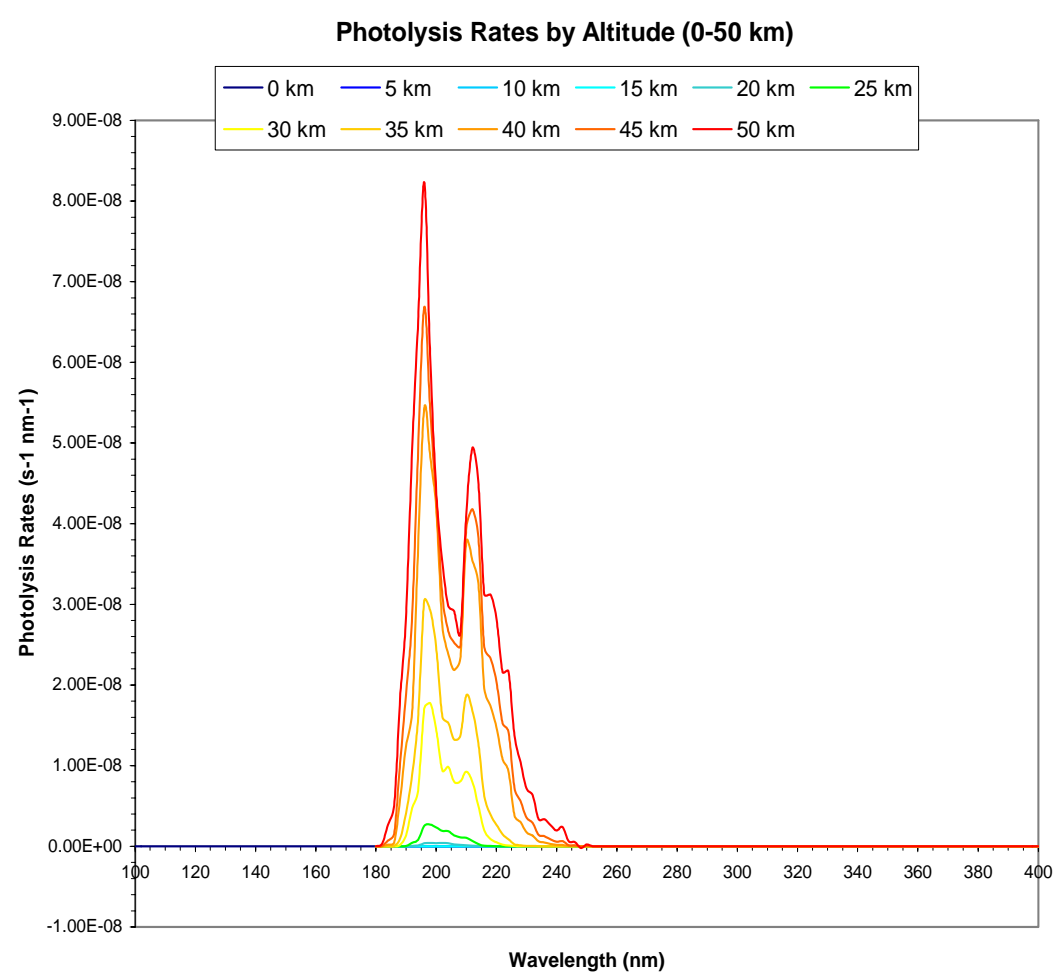

Fig. (6) Photolysis rates (by wavelength and altitude) for Vinyl Chloride

The reciprocal of the total photolysis rate gives the local photolysis lifetime at that altitude, i.e. the time taken for the molecule to photodissociate if altitude and solar actinic flux remain constant.

The results show that the local photolysis lifetime of this molecule varies and molecular photo - absorption cross-section at different altitudes and wavelengths assuming that the quantum yield for photodissociation being unity, at wavelength $196 \mathrm{~nm}$, the lifetime will then be nearly 4 days (3.99 days) at altitude $1000 \mathrm{~km}$. 


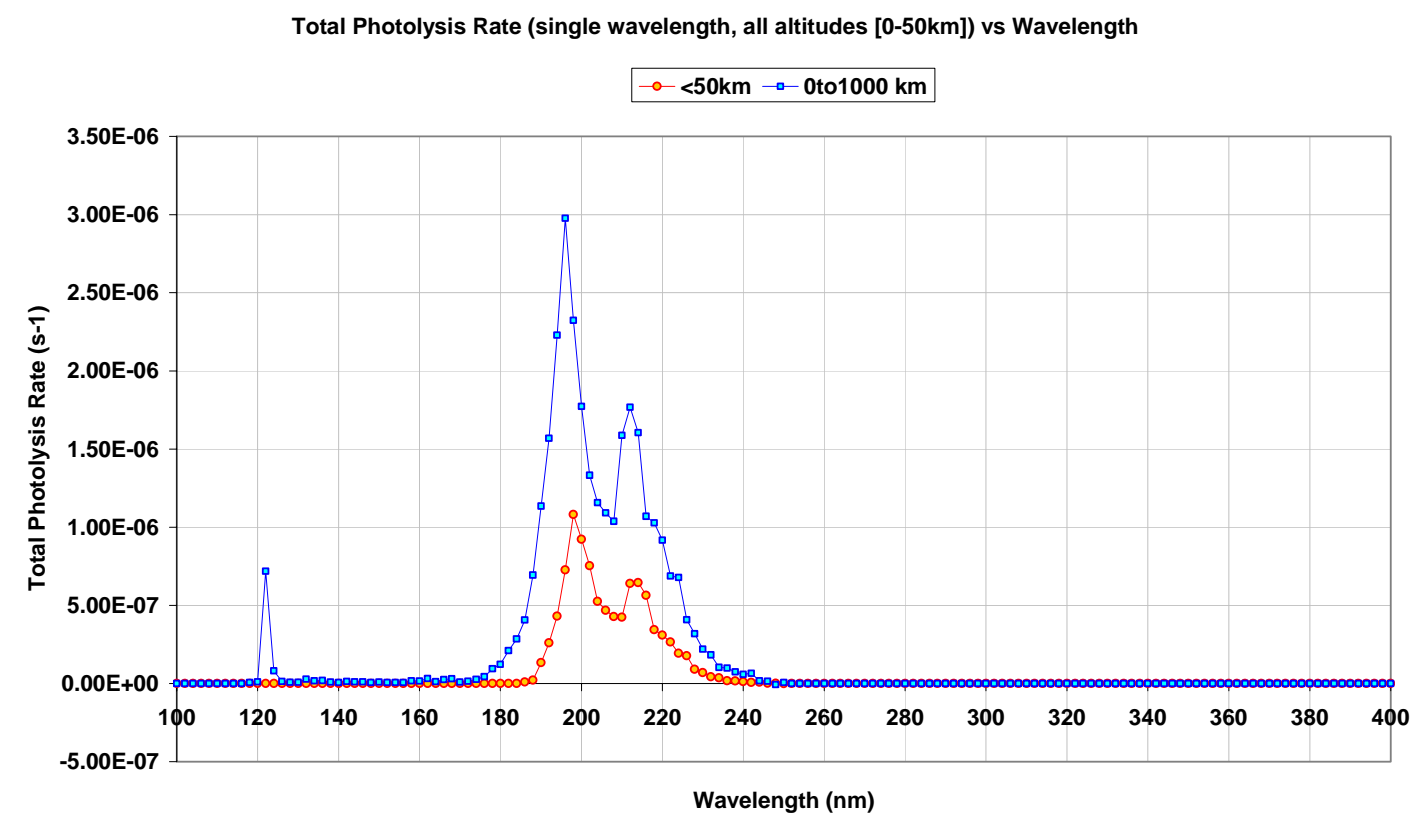

Fig. (7) Photolysis rate of ( 50 and $1000 \mathrm{~km}$ ) for Vinyl Chloride

The lifetimes calculated are for molecules with fixed altitude in a sunlit, clear sky atmosphere. Fig. (7) Represent the relationship between photolysis rate and wavelength. At wavelength $198 \mathrm{~nm}$ and altitude 50 $\mathrm{km}$, the lifetime is 10.7 days. It is these long lifetimes at low altitudes coupled with its strong infrared absorption properties that make these molecules such a strong greenhouse gases.

\section{Conclusion}

The experimental results presented in this paper provide the highest resolution VUV photo - absorption spectra of Vinyl Chloride in the range $(5-11) \mathrm{eV}(115>\lambda>250) \mathrm{nm}$, yet reported. Rydberg states were assigned for the first time. Photolysis rates and local lifetimes of the molecules were calculated for various altitudes in the atmosphere.

\section{Acknowledgements}

The author wish to acknowledge the college of Science -Mosul University for supporting the fellowship to verify this work at UK. 


\section{References}

1. Yit-Tsong Chena, "Molecular Rydberg States and Ionization Energy Studied by Two-Photon Resonant Ionization Spectroscopy "Jour nal of the Chinese Chemical Society, 2002, 49, 703-722

2. Budavari S., O’Neil M., Smith A., Heckelman P. and Kinneary J. The Merck index, an encyclopaedia of chemicals, drugs, and biological; $12^{\text {th }}$ edition, 1996.

3. Jia-lin Chang, Runhua Li, Jen-Chieh Wu, Jau-Chin Shieh, and YitTsong Chena ; "Two-photon vibronic spectra of vinyl chloride at 7.3-10 eV" Journal of Chemical Physics vol. 115, No. 131 OCTOBER 2001

4. Jia-Lin Chang and Yit-Tsong Chena; "Ab initio calculations of low-lying electronic states of vinyl chloride" Journal of Chemical Physics vol. 116, No. 171 MAY 2002

5. Robin, M. B. "Higher Excited States of Polyatomic Models ", 1974, Vol.1, Accadimic press.

6. C. S. C. de Castro, A. C. P. de Azevedo, E. E. C. Monteiro, R. R. Pinho and G. G. B. de Souza GAS EMISSION FROM CHLORINATED POLYMERS INDUCED BY SYNCHROTRON RADIATION 1997.

7. Eden, Samuel; 2003, thesis, University of London, dept. of Physic. and Astronomy.

8. Kendall, P. A. Thesis , University of London " Spectroscopic Studies of Atmospheric Molecules Related to Globale Warming, 2003.

9. International chemical safety cards (WHO/IPSO/ILO), 1999.

10. Determination of absolute photo ionization cross sections for vinyl and propargyl radicals Jason C. Robinson, Niels E. Sveum, and Daniel M. Neumark Journal of Chemical Physics Volume 119, Number 11 - 15 September 2003. 